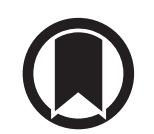

CrossMark

\title{
Pneumonia versus graft dysfunction as the cause of acute respiratory failure after lung transplant: a 4-year multicentre prospective study in 153 adults requiring intensive care admission
}

\author{
Cristopher Mazo 1,2,3,4 , Teresa Pont (10 ${ }^{1,2,3}$, Maria A. Ballesteros ${ }^{5}$, Eloísa López ${ }^{6}$ \\ Luzdivina Rellán ${ }^{7}$, Juan C. Robles ${ }^{8}$ and Jordi Rello ${ }^{2,3}$
}

Affiliations: ${ }^{1}$ Transplant Procurement Dept, Vall d'Hebron University Hospital, Barcelona, Spain. ${ }^{2}$ Vall d'Hebron Research Institute, Barcelona, Spain. ${ }^{3}$ CIBERES, Instituto de Salud Carlos III, Madrid, Spain. ${ }^{4}$ Dept of Medicine, Universitat Autònoma de Barcelona, Barcelona, Spain. ${ }^{5}$ Intensive Care Medicine Dept, Marqués de Valdecilla University Hospital, Santander, Spain. ${ }^{6}$ Intensive Care Medicine Dept, 12 de Octubre University Hospital, Madrid, Spain. ${ }^{7}$ Intensive Care Medicine Dept, A Coruña University Hospital, A Coruña, Spain. ${ }^{8}$ Intensive Care Medicine Dept, Reina Sofia University Hospital, Córdoba, Spain.

Correspondence: Cristopher Mazo, Transplant Procurement Dept, Vall d'Hebron University Hospital, Passeig de la Vall d'Hebron 119-129, Edificio Materno-Infantil 11ª Planta, 08035 Barcelona, Spain. E-mail: cmazođvvebron.net

@ERSpublications

In lung transplant adults, pneumonia causes most ICU readmissions and independently influences risk of death, as chronic lung allograft dysfunction also does, particularly the restrictive phenotype. However, acute allograft dysfunction is far less common http://bit.ly/2Xvitv5

Cite this article as: Mazo C, Pont T, Ballesteros MA, et al. Pneumonia versus graft dysfunction as the cause of acute respiratory failure after lung transplant: a 4-year multicentre prospective study in 153 adults requiring intensive care admission. Eur Respir J 2019; 54: 1801512 [https://doi.org/10.1183/13993003.01512-2018].

ABSTRACT We aimed to assess the main causes of intensive care unit (ICU) readmissions in lung transplant adults and to identify independent predictors of ICU mortality (primary end-point).

This Spanish five-centre prospective cohort study enrolled all lung transplant adults with ICU readmissions after post-transplant ICU discharge between 2012 and 2016. Patients were followed until hospital discharge or death.

153 lung transplant recipients presented 174 ICU readmissions at a median (interquartile range) of 6 (2-25) months post-transplant. Chronic lung allograft dysfunction was reported in 39 (25.5\%) recipients, 13 of whom (all exitus) had restrictive allograft syndrome (RAS). Acute respiratory failure (ARF) (110 (71.9\%)) was the main condition requiring ICU readmission. Graft rejection (six (5.4\%) acute) caused only 12 (10.8\%) readmissions whereas pneumonia $(56(36.6 \%))$ was the main cause (50 admitted for ARF and six for shock), with Pseudomonas aeruginosa (50\% multidrug resistant) being the predominant pathogen. 55 (35.9\%) and 69 (45.1\%) recipients died in the ICU and the hospital, respectively. Bronchiolitis obliterans syndrome (BOS) stage 2 (adjusted OR (aOR) 7.2 (95\% CI 1.0-65.7)), BOS stage 3 (aOR 13.7 (95\% CI 2.5-95.3)), RAS $(\mathrm{aOR}>50)$ and pneumonia at ICU readmission (aOR 2.5 (95\% CI 1.0-7.1)) were identified in multivariate analyses as independent predictors of ICU mortality. Only eight (5.2\%) patients had positive donor-specific antibodies prior to ICU readmission and this variable did not affect the model.

ARF was the main condition requiring ICU readmission in lung transplant recipients and was associated with high mortality. Pneumonia was the main cause of death and was also an independent predictor. RAS should receive palliative care rather than ICU admission.

This article has supplementary material available from erj.ersjournals.com

Received: 09 Aug 2018 | Accepted after revision: 25 June 2019

Copyright OERS 2019 


\section{Introduction}

Lung transplant has shown improved survival, although the median survival is only 5.8 years [1]. The number of lung transplants and related intensive care unit (ICU) readmissions has risen steadily in recent years $[2,3]$. The fact that lung transplant subjects require more aggressive immunosuppression than other transplant recipients has obvious implications [4]. Interestingly, both lung infections and graft dysfunction present as acute respiratory failure (ARF) [5], and are the leading causes of death within the first year [2, $6,7]$. This means that management of ARF is a challenge: in the case of infection it requires a decrease in immunosuppression and in the case of graft dysfunction, the exact opposite (i.e. an increase). Long-term survival depends mainly on the avoidance of chronic lung allograft dysfunction (CLAD) [8], which includes different phenotypes and stages [9-11]. Moreover, additional concerns, which also affect physicians not familiar with lung transplantion, include whether CLAD should influence ICU readmission criteria, what diagnostic workup should be performed, what empirical antimicrobial, antifungal and antiviral coverage should be added, whether immunoglobulin should be administered, and whether techniques such as plasmapheresis should be implemented.

$\mathrm{ARF}$ and sepsis have been described as the most frequent conditions determining ICU readmissions in lung transplant recipients [12-17]. However, factors influencing ICU readmissions in this unique subgroup of immunocompromised patients after the post-operative period have been reported by only a few studies, mainly with retrospective designs and conducted at single centres (supplementary table S1). Thus, it is of paramount importance to have an updated snapshot of this emerging condition. The main objectives of this study were to assess the principal causes of ICU readmissions in adult lung transplant recipients, and to identify potential predictors of ICU (primary end-point) and hospital mortality.

\section{Methods}

\section{Study design}

A multicentre, prospective, observational study was carried out (August 2012-June 2016) at five Spanish tertiary care university hospitals with well-established lung transplant programmes. Overall, the sample represents $73.2 \%$ of lung transplants performed in Spain during the study period (supplementary table S2).

\section{Study population}

The study population comprised all consecutive adult lung transplant recipients who required ICU readmission ( $>7$ days after ICU discharge post-transplant) during the study period (4 years). Subjects were identified at the time of ICU readmission. Criteria for ICU readmission included requirement of invasive mechanical ventilation (IMV) or noninvasive mechanical ventilation and/or invasive haemodynamic monitoring. Patients were followed until hospital discharge or death. Immunosuppressive and prophylaxis regimens are detailed in supplementary appendix S1. For patients with multiple admissions, only data from the first episode were studied.

\section{Data collection}

The data collected were age, sex, pre-transplant diagnosis, type of lung transplant (single or bilateral), time since lung transplant at ICU readmission, forced expiratory volume in $1 \mathrm{~s}(\mathrm{FEV} 1)$ and forced vital capacity (FVC), donor-specific antibody (DSA) status before ICU readmission, histopathological pattern and C4d immunostaining in transbronchial biopsies (TBBs), history of acute rejection and CLAD (including the different phenotypes and severity), indication for ICU readmission, Acute Physiology and Chronic Health Evaluation (APACHE) II score at $24 \mathrm{~h}$ after ICU readmission, Sequential Organ Failure Assessment (SOFA) score at ICU readmission, source and aetiology of infection, requirement and length of IMV, requirement of vasopressors, length of ICU/hospital stay, ICU/hospital mortality, and cause of death. Management data on the immediate perioperative period were not recorded.

\section{Pneumonia}

Pneumonia was defined as a new radiographic infiltrate, $\geqslant 10^{4} \mathrm{CFU} \cdot \mathrm{mL}^{-1}$ (for nonopportunistic pathogens) in bronchoalveolar lavage (BAL) fluid and at least two of the following conditions: fever $>38.5^{\circ} \mathrm{C}$, leukocyte count $<4000$ or $>11000 \mathrm{~mm}^{-3}$ and purulent secretions [18]. Standard empirical antibiotics consisted of meropenem plus amikacin, adjusted on antibiotic susceptibility testing. Standard diagnostic workup involved blood cultures, BAL (with TBB) for standard cultures to identify conventional bacterial pathogens, including Nocardia spp., and filamentous fungi. Multiplex PCR for 13 respiratory viruses was performed in respiratory specimens. Searches for Pneumocystis jirovecii, PCR for cytomegalovirus (CMV) and galactomannan detection were performed in BAL specimens (supplementary appendix S1). 


\section{Definitions and differential diagnosis}

Indications for ICU readmission were categorised as ARF, shock, post-operative care and others. ARF was defined as arterial oxygen tension $<60 \mathrm{mmHg}$ or arterial oxygen saturation measured by pulse oximetry $<90 \%$ on room air, or tachypnoea $>30 \mathrm{~min}^{-1}$, respiratory distress, dyspnoea at rest or cyanosis. ARF differential diagnosis included pneumonia, acute cellular rejection, antibody-mediated rejection (AMR), exacerbation of CLAD, acute pulmonary oedema, intra-alveolar bleeding, atelectasis and mechanical complications. Diagnostic workup is detailed in supplementary appendix S2. Diagnoses at ICU readmission were categorised as pneumonia, acute pulmonary oedema, bronchial stenosis, acute cellular rejection, AMR and others. Acute cellular rejection was defined based on TBB, evaluated by a lung transplant pathologist and graded according to the 2007 International Society for Heart and Lung Transplantation (ISHLT) guidelines [19]. TBB was performed for early detection of allograft rejection, unless contraindicated by ARF severity, after performing BAL. Three to six biopsies were taken from the lower and middle lobe of the right lung or from the lower lobe and lingula of the left lung. AMR was defined as graft dysfunction, confirmed presence of DSAs in serum [19] and pathology compatible with AMR [20]. Sepsis and septic shock were defined based on the Sepsis 2 criteria [21]. Primary graft dysfunction and its grade were determined using the ISHLT definition [22]. Patients were classified as having CLAD if they met ISHLT criteria [23]. Antimicrobial susceptibility of isolates was tested using disc diffusion and resistant strains were checked using the gradient diffusion method. Minimum inhibitory concentrations (MICs) were classified using the European Committee on Antimicrobial Susceptibility Testing breakpoints [24]. Multidrug resistance and extensive drug resistance have been reported elsewhere [25]. Acute kidney injury was determined using the Second International Consensus of the Acute Dialysis Quality Initiative [26]. Chronic kidney disease (CKD) was defined as kidney damage or glomerular filtration rate $<60 \mathrm{~mL} \cdot \mathrm{min}^{-1} \cdot 1.73 \mathrm{~m}^{-2}$ for $\geqslant 3$ months, irrespective of cause, estimated from calibrated serum creatinine and estimating equations such as the Modification of Diet in Renal Disease study equation or the Cockcroft-Gault formula [27].

\section{Statistical analysis}

Continuous variables were described as median (interquartile range (IQR)) and categorical variables as counts (percentages). Several pairs of clinical variables were compared using Pearson and Spearman correlations. Patients were categorised according to their status (i.e. survivors and nonsurvivors from the ICU and the hospital). Clinical variables in both groups of patients were compared using the Chi-squared test for categorical variables (or Fisher's exact test if absolute frequency was $<5$ ) and the Mann-Whitney U-test for quantitative variables. p-values were adjusted using the Benjamini-Hochberg method to deal with problems of multiple comparisons deriving from multiple tests.

To analyse the predictive factors of ICU mortality (primary end-point) and hospital mortality (secondary end-point), logistic regression models were used. The logistic regression model methodology is shown in supplementary appendix A3. Kaplan-Meier curves were developed using the log-rank test. All analyses were performed in $\mathrm{R}$ version 3.4.1 (www.R-project.org).

\section{Ethics}

The study was approved by the Ethics Committee of Vall d'Hebron University Hospital (Barcelona, Spain) (PR(AG)101/2012). The need for consent was waived due to the observational nature of the study.

\section{Results}

153 lung transplant recipients (68.6\% male, median (IQR) age 58 (50-63) years, 53.6\% bilateral lung transplant) requiring 174 ICU readmissions (15 patients with two readmissions and three patients with three readmissions) were enrolled at a median (IQR) of 6 (2-25) months after lung transplant. 33 (21.6\%) patients were readmitted within the first month following lung transplant (20 due to ARF and two due to sepsis), 53 (34.7\%) within 3 months, 73 (47.8\%) within 6 months, 85 (55.6\%) within 1 year, $113(73.9 \%)$ within 18 months and 40 (26.1\%) spanning a range of 19-192 months. The distribution and demographics of lung transplant recipients and ICU readmissions by hospital are detailed in tables 1 and 2.95 (62.1\%) and $40(26.1 \%)$ recipients underwent intubation and vasopressor treatment within $24 \mathrm{~h}$ of admission, respectively. Fewer than $2 \%$ had missing data. 39 (25.5\%) had CLAD. Pulmonary fibrosis and COPD were the most common pre-transplant diseases. Phrenic paresis, primary graft dysfunction and acute kidney injury were the most frequent early complications (in the immediate post-operative period); the most common late complications (after ICU discharge) were bacterial infections, followed by CLAD, history of acute rejection episodes and CKD. Moreover, 88 (57.7\%) patients developed at least one ICU complication.

79 subjects underwent TBB. DSAs (anti-HLA alloantibodies) were positive in eight (5.2\%) patients before ICU readmission. Graft rejection (six $(5.4 \%)$ acute) was the cause of readmission in only $12(10.8 \%)$ recipients. Differences between each year during the study period are reported in supplementary table S3. 
TABLE 1 Demographic and lung transplant data of 153 patients readmitted to five intensive care units (ICUs) in Spain

$\begin{array}{ccccc}\begin{array}{c}\text { Vall d'Hebron } \\ \text { University } \\ \text { Hospital } \\ \text { (Barcelona) }\end{array} & \begin{array}{c}\text { Marqués } \\ \text { de Valdecilla } \\ \text { University } \\ \text { Hospital } \\ \text { (Santander) }\end{array} & \begin{array}{c}\text { A Coruña } \\ \text { University } \\ \text { Hospital } \\ \text { (A Coruña) }\end{array} & \begin{array}{c}12 \text { de Octubre } \\ \text { University } \\ \text { Hospital } \\ \text { (Madrid) }\end{array} & \begin{array}{c}\text { Reina Sofia } \\ \text { University } \\ \text { Hospital } \\ \text { (Córdoba) }\end{array} \\ & & & \end{array}$

\begin{tabular}{|c|c|c|c|c|c|c|}
\hline Patients & 77 (50.3) & $40(26.1)$ & $17(11.1)$ & $14(9.2)$ & 5 (3.3) & $153(100.0)$ \\
\hline Age years & $57(49-62)$ & $57.5(52-64)$ & $58(56-62)$ & $61(55.8-64.5)$ & $19(17-40)$ & $58(50-63)$ \\
\hline Male & $54(70.1)$ & 22 (55.0) & $14(82.4)$ & 12 (85.7) & $3(60.0)$ & $105(68.6)$ \\
\hline \multicolumn{7}{|c|}{ Pre-transplant lung disease } \\
\hline COPD & $22(28.6)$ & $16(40.0)$ & 6 (35.3) & $6(42.9)$ & $1(20.0)$ & $51(33.4)$ \\
\hline Cystic fibrosis & $6(7.8)$ & $3(7.5)$ & 0 & 0 & $3(60.0)$ & $12(7.8)$ \\
\hline DILD & 3 (3.9) & $1(2.5)$ & $2(11.8)$ & $2(14.3)$ & 0 & $8(5.2)$ \\
\hline Other $\#$ & $7(9.1)$ & $3(7.5)$ & $1(5.9)$ & 0 & 0 & $11(7.2)$ \\
\hline \multicolumn{7}{|l|}{ Type of lung transplant } \\
\hline Bilateral & $42(54.5)$ & $22(55.0)$ & $6(35.3)$ & $8(57.2)$ & $4(80.0)$ & $82(53.6)$ \\
\hline Left & $26(33.8)$ & $9(22.5)$ & $3(17.6)$ & $2(14.3)$ & 0 & $40(26.1)$ \\
\hline Right & $9(11.7)$ & $9(22.5)$ & $8(47.1)$ & $4(28.5)$ & $1(20.0)$ & $31(20.3)$ \\
\hline \multicolumn{7}{|c|}{ History of early " complications post-transplant ${ }^{\S}$} \\
\hline Gastroparesis & $15(19.5)$ & $2(5.0)$ & $4(23.5)$ & 1 & 0 & $22(14.4)$ \\
\hline Tracheobronchitis & $14(18.2)$ & $1(2.5)$ & 0 & $1(7.1)$ & 0 & $16(10.5)$ \\
\hline Haemorrhage & $7(9.1)$ & $5(12.5)$ & 0 & $1(7.1)$ & 0 & $13(8.5)$ \\
\hline Thrombosis & $4(5.2)$ & $6(15.0)$ & 0 & $2(14.3)$ & 0 & $12(7.8)$ \\
\hline CMV replication & $9(11.7)$ & $1(2.5)$ & 0 & 0 & 0 & $10(6.5)$ \\
\hline Suture stenosis & $5(6.5)$ & $1(2.5)$ & 0 & 0 & 0 & $6(3.9)$ \\
\hline Suture dehiscence & $4(5.2)$ & 0 & 0 & 0 & 0 & $4(2.6)$ \\
\hline \multicolumn{7}{|c|}{ History of late ${ }^{+}$complications post-transplant ${ }^{\S}$} \\
\hline Infection & $20(26.0)$ & $16(40.0)$ & $2(11.8)$ & $3(21.4)$ & 0 & $41(26.8)$ \\
\hline CLAD & $23(29.9)$ & $7(17.5)$ & $5(29.4)$ & $3(21.4)$ & $1(20.0)$ & $39(25.5)$ \\
\hline Restrictive CLAD & $10(13.0)$ & $2(5.0)$ & $1(5.9)$ & 0 & 0 & $13(8.5)$ \\
\hline Obstructive CLAD & $13(16.9)$ & $5(12.5)$ & $4(23.5)$ & $3(21.4)$ & $1(20.0)$ & $26(17.0)$ \\
\hline BOS stage 1 & $4(5.2)$ & $3(7.5)$ & 2 (11.8) & 2 (14.3) & 0 & $11(7.2)$ \\
\hline
\end{tabular}

Data are presented as $\mathrm{n}(\%)$ or median (interquartile range); percentages may not total $100 \%$ due to rounding. IPF: idiopathic pulmonary fibrosis; COPD: chronic obstructive pulmonary disease; DILD: diffuse interstitial lung disease; PAH: pulmonary arterial hypertension; PGD: primary graft dysfunction; AKI: acute kidney injury; CMV: cytomegalovirus; CLAD: chronic lung allograft dysfunction; BOS: bronchiolitis obliterans syndrome; ARE: acute rejection episode; CKD: chronic kidney disease. ${ }^{\#}$ : categories with one patient; ${ }^{\text {ๆ}}$ : immediate post-operative period; ${ }^{+}$: more than one complication in some readmissions; ${ }^{\S}$ : after ICU discharge.

Infection was present in $75(49.0 \%)$ patients (table 2). At ICU readmission, 55 presented with sepsis and 40 presented with septic shock criteria. 39 instances of sepsis were secondary to respiratory tract infections, followed by six intra-abdominal (one Clostridium difficile infection), four urinary tract, four catheter-related and two of unknown site.

Pneumonia was the most common 1) diagnosis among ICU readmissions (56 out of $153(36.6 \%)$ ), 2) cause of ARF (50 out of 110 (45.5\%)), 3) indication for IMV (39 out of 95 (41.1\%)), and 4) cause of sepsis (39 out of $55(67.3 \%)$ ) and septic shock (28 out of $40(70.0 \%)$ ) (figure 1). Most pneumonia ICU 
TABLE 2 Characteristics of 153 lung transplant patients readmitted to five intensive care units (ICUs) in Spain

\begin{tabular}{|c|c|c|c|c|c|c|}
\hline & $\begin{array}{l}\text { Vall d'Hebron } \\
\text { University } \\
\text { Hospital } \\
\text { (Barcelona) }\end{array}$ & $\begin{array}{l}\text { Marqués } \\
\text { de Valdecilla } \\
\text { University } \\
\text { Hospital } \\
\text { (Santander) }\end{array}$ & $\begin{array}{l}\text { A Coruña } \\
\text { University } \\
\text { Hospital } \\
\text { (A Coruña) }\end{array}$ & $\begin{array}{l}12 \text { de Octubre } \\
\text { University } \\
\text { Hospital } \\
\text { (Madrid) }\end{array}$ & $\begin{array}{l}\text { Reina Sofia } \\
\text { University } \\
\text { Hospital } \\
\text { (Córdoba) }\end{array}$ & Total \\
\hline Patients & $77(50.3)$ & $40(26.1)$ & $17(11.1)$ & $14(9.2)$ & 5 (3.3) & $153(100.0)$ \\
\hline $\begin{array}{l}\text { Time after lung transplant at ICU } \\
\text { readmission months }\end{array}$ & $8(3.0-31.0)$ & $4.5(1.0-16.2)$ & $3(1.0-6.0)$ & $15.5(4.3-29)$ & $4(1.0-41.0)$ & $6(2.0-25.0)$ \\
\hline SOFA score & $5(4.0-9.0)$ & $3(1.0-6.8)$ & $4(3.0-7.0)$ & $6(3.3-7.0)$ & $7(5.0-8.0)$ & $5(3.0-8.0)$ \\
\hline Last FEV1 $\%$ pred & $44(16.0-60.0)$ & $42.5(21.8-64.5)$ & $44(23.0-65.0)$ & $57(49.5-62.0)$ & $55(48.0-61.0)$ & $42(16.0-58.0)$ \\
\hline Last FVC \% pred & $48(29.0-62.0)$ & $52(43.0-74.0)$ & $47(28.0-60.0)$ & $74(55.0-80.8)$ & $50(29.0-69.0)$ & $46(29.0-65.0)$ \\
\hline \multicolumn{7}{|l|}{ Indication for ICU readmission } \\
\hline ARF & 58 (75.3) & $26(65.0)$ & $14(82.4)$ & $10(71.5)$ & $2(40.0)$ & $110(71.9)$ \\
\hline Shock & $3(3.9)$ & $2(5.0)$ & $1(5.9)$ & $2(14.3)$ & $1(20.0)$ & 9 (5.9) \\
\hline Infection & $37(48.1)$ & $24(60.0)$ & $8(47.1)$ & $5(47.1)$ & $1(20.0)$ & $75(49.0)$ \\
\hline Pneumonia & $29(37.7)$ & $16(40.0)$ & 6 (35.3) & $4(28.5)$ & $1(20.0)$ & $56(36.6)$ \\
\hline Sepsis & $28(36.4)$ & 19 (47.5) & $4(23.5)$ & $3(30.0)$ & $1(20.0)$ & 55 (35.9) \\
\hline Septic shock & $20(26.0)$ & 13 (32.5) & $4(23.5)$ & $3(30.0)$ & $1(20.0)$ & $40(26.1)$ \\
\hline \multicolumn{7}{|l|}{ Causes of ARF at ICU readmission } \\
\hline Pneumonia & $26(44.8)$ & $14(53.9)$ & $5(35.8)$ & $4(40.0)$ & $1(50.0)$ & $50(45.5)$ \\
\hline Acute pulmonary oedema & $4(6.9)$ & $2(7.7)$ & $1(7.1)$ & $1(10.0)$ & 0 & $8(7.3)$ \\
\hline Bronchial stenosis & $1(1.7)$ & $2(7.7)$ & $4(28.6)$ & 0 & 0 & $7(6.4)$ \\
\hline Acute rejection & $3(5.2)$ & $2(7.7)$ & $1(7.1)$ & 0 & 0 & $6(5.4)$ \\
\hline Exacerbation of CLAD & $3(5.2)$ & $1(3.8)$ & $1(7.1)$ & 0 & $1(50.0)$ & $6(5.4)$ \\
\hline Other ${ }^{+}$ & $21(36.2)$ & $5(19.2)$ & $2(14.3)$ & $5(35.7)$ & 0 & $33(30.0)$ \\
\hline \multicolumn{7}{|l|}{ Techniques and support } \\
\hline Other ${ }^{\#}$ & 0 & $2(15.4)^{+}$ & $1(20.0)^{+}$ & 0 & 0 & $3(5.5)^{+}$ \\
\hline Deaths in hospital & $38(48.1)$ & $17(42.5)$ & $9(58.8)$ & 4 (33.3) & $1(20.0)$ & $69(45.1)$ \\
\hline
\end{tabular}

Total

Data are presented as $n(\%)$ or median (interquartile range); percentages may not total $100 \%$ due to rounding. APACHE: Acute Physiology and Chronic Health Evaluation; SOFA: Sequential Organ Failure Assessment; FEV1: forced expiratory volume in 1 s; FVC: forced vital capacity; ARF: acute respiratory failure; CLAD: chronic lung allograft dysfunction; IMV: invasive mechanical ventilation; FBC: fibreoptic bronchoscopy. ${ }^{\#}$ : categories with one patient; ${ }^{\text {9: }}$ : admission diagnoses are not mutually exclusive; ${ }^{+}$: proportion of ICU deaths.

readmissions were admitted directly from the emergency department. Only $47.3 \%$ of the patients with pneumonia survived to ICU discharge (figure 2). Among pneumonia patients, ICU survival was $71.4 \%$ in the subgroup who did not require IMV or vasopressors, $42.9 \%$ in the subgroup who required only IMV, and only $28.0 \%$ in the subgroup requiring both IMV and vasopressors (figure 2). 32 out of 71 (45.1\%) single lung transplant subjects had pneumonia, compared with 24 out of $82(29.3 \%)$ with bilateral lung transplant ( $\mathrm{p}>0.05) ; 14$ out of $32(43.8 \%)$ single lung transplant subjects survived to ICU readmission due to pneumonia compared with 12 out of 24 (50.0\%) with bilateral lung transplant ( $p>0.05) .46(82.1 \%)$ organisms were isolated from 56 patients with pneumonia, including Pseudomonas aeruginosa in 23 patients (50.0\%) (15 out of 23 (65.2\%) had positive cultures for P. aeruginosa prior to ICU readmission), $14(60.7 \%)$ of them multidrug resistant; most $P$. aeruginosa were only susceptible to amikacin and colistin, with a MIC50 breakpoint $>8 \mathrm{mg} \cdot \mathrm{dL}^{-1}$ to meropenem. Prior antimicrobial exposure was universal in this cohort of ICU subjects, with exposure to penicillins in $58.7 \%$ (38.5\% to cephalosporins and $36.5 \%$ to 


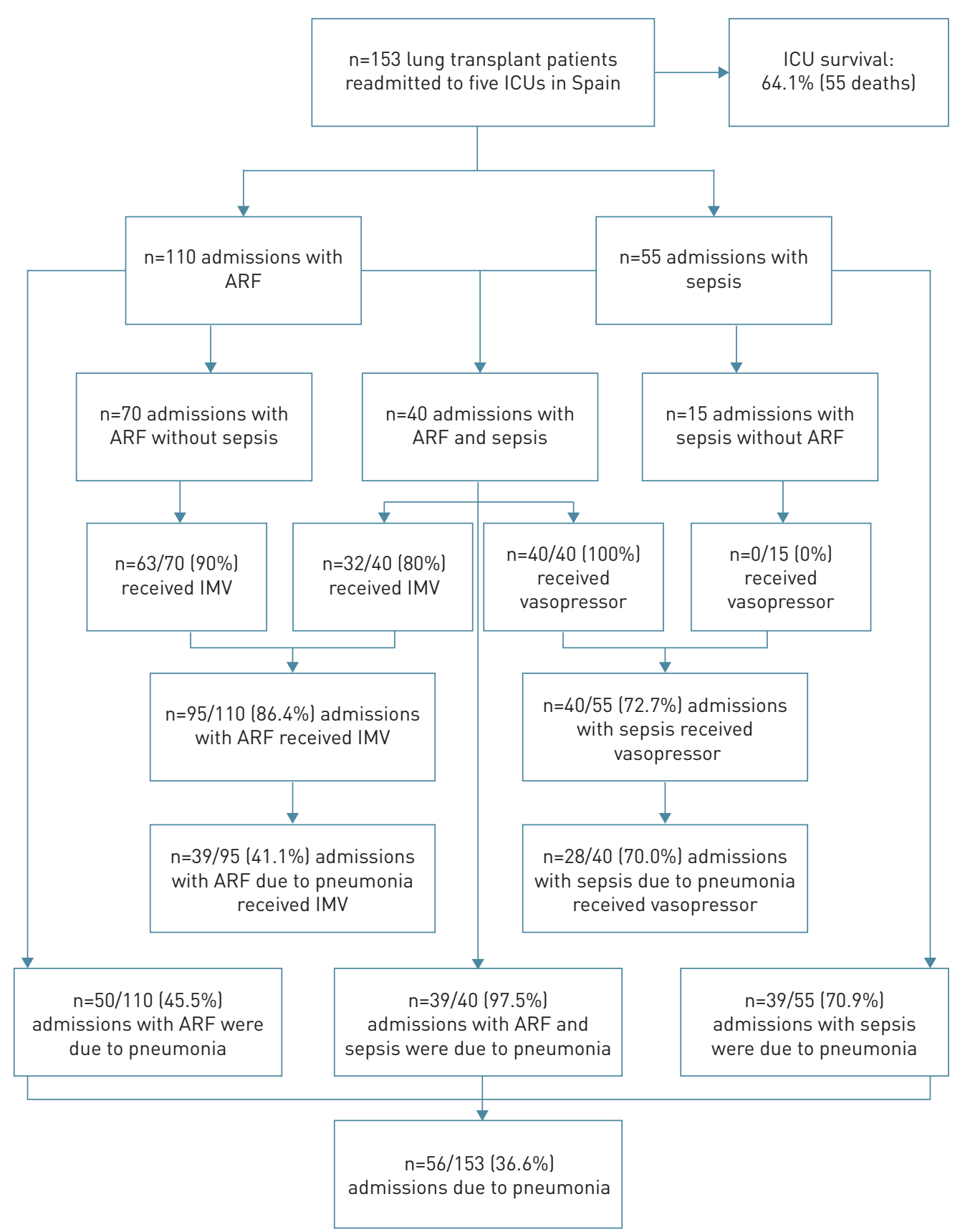

FIGURE 1 Study flowchart. ICU: intensive care unit; ARF: acute respiratory failure; IMV: invasive mechanical ventilation.

carbapenems). Additionally, all patients were exposed to colistin due to selective digestive decontamination during the post-operative period. Gram-positive cocci were isolated in respiratory specimens from 10 patients (21.6\%), Enterobacteriaceae from eight (17.3\%), Aspergillus spp. from three (6.5\%) and influenza A virus from two (4.6\%). Figure 3 and supplementary figure S2 show the chronology of the aetiology of the ICU readmissions and the pattern of susceptibility of $P$. aeruginosa (multidrug resistant versus susceptible) due to pneumonia according to time after lung transplant, respectively. No CMV infection was identified as the cause of ICU readmission.

55 (35.9\%) subjects died in the ICU (with life-sustaining therapies being withdrawn from $23(41.8 \%)$ subjects). Main mortality causes were multiorgan failure (49.1\%) and hypoxaemia (45.5\%), with most deaths occurring within the first month (figure 4). Among 13 recipients with RAS, ICU survival was $7.7 \%$ (none were discharged home alive) compared with $53.8 \%(\mathrm{p}<0.05)$ in patients with BOS, in whom survival correlated inversely with severity of obstruction (table 3 ). 


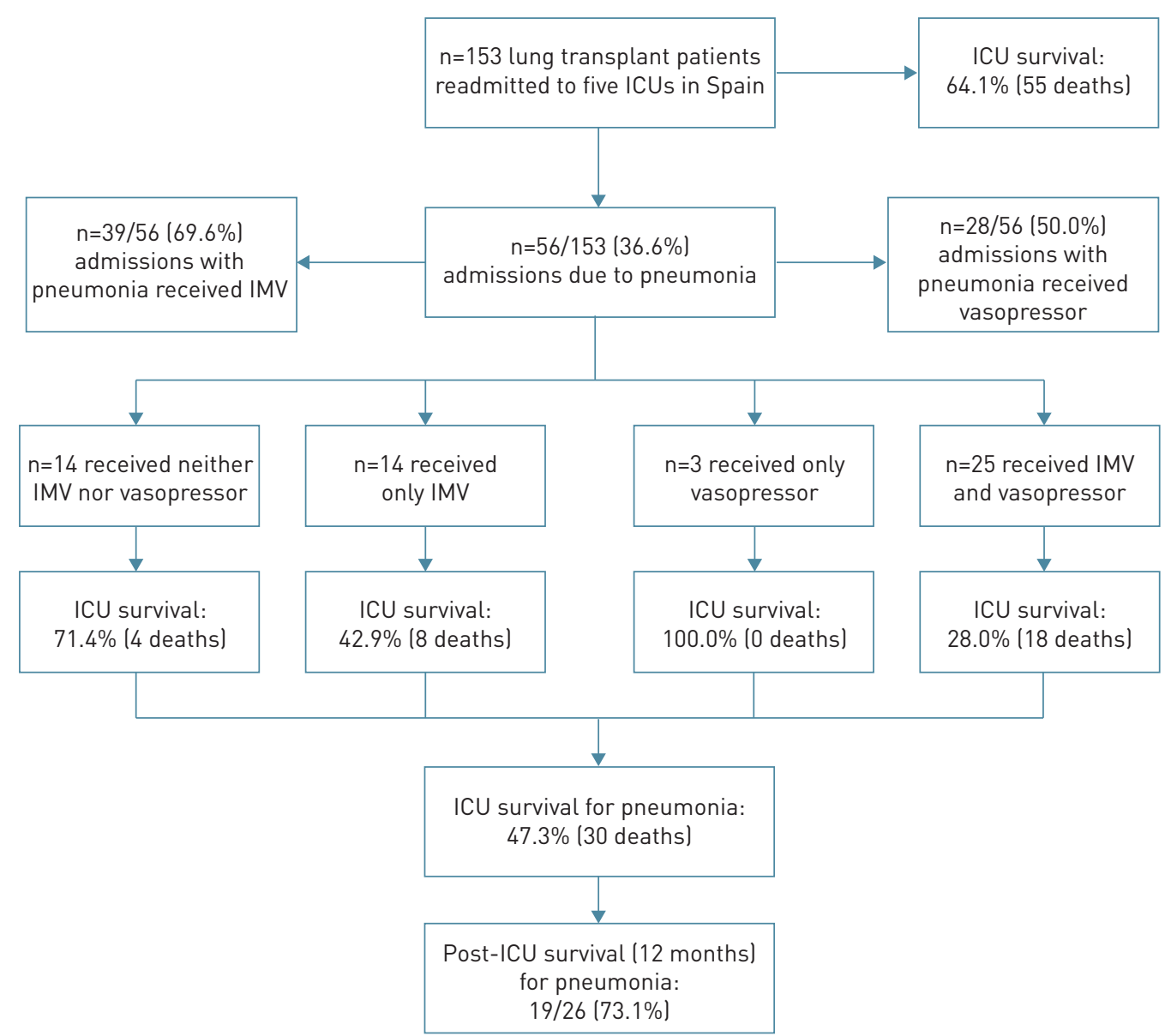

FIGURE 2 Flowchart of pneumonia cases. ICU: intensive care unit; IMV: invasive mechanical ventilation.

After ICU discharge, 14 patients died while still hospitalised, with a median (IQR) post-ICU length of stay of 26 (18-169) days (12-month survival post-ICU 90.9\%). Finally, overall hospital mortality was 69 (45.1\%) patients. Despite the fact that patients diagnosed with ARF, sepsis or septic shock at ICU readmission (the most common ICU readmission indications) had low ICU survival (61.4\% in ARF patients, $50.0 \%$ in sepsis and $38.5 \%$ in septic shock), these conditions were not associated with ICU mortality in the multivariate analysis. Interestingly, ICU survival in pneumonia patients fell to $48.0 \%$ in cases with ARF, $43.6 \%$ in cases with sepsis and $35.7 \%$ in cases with septic shock. The 12 -month survival post-ICU in pneumonia subjects was $73.1 \%$ compared with $97.2 \%$ for other conditions $(\mathrm{p}<0.001)$. Among pneumonia patients, 23 with a causative agent died compared with seven with unknown cause (50\% versus $70 \%$; $>0.05$ ), whereas the median ICU length of stay for survivors did not differ (data not shown). Survival in CLAD patients with ARF was $31.3 \%$. The variables associated with ICU mortality on univariate analysis were APACHE II score $>20$, SOFA score $>6$, IMV and vasopressor requirement, pre-admission diagnosis of CLAD (obstructive/restrictive phenotypes), CKD, acute rejection episode, osteoporosis, ICU readmission $>1$ month after lung transplant, and pneumonia; these variables were selected for multivariate analyses (table 3). In the resulting model (table 4), adjusted for centre, IMV (adjusted OR (aOR) 8.2 (95\% CI 2.6-34.7)), vasopressors (aOR 3.6 (95\% CI 1.2-11.1)), pneumonia (aOR 2.5 (95\% CI 1.0-7.1)), and pre-admission diagnosis of BOS stage 2, stage 3 and RAS (aOR 7.2 (95\% CI 1.0-65.7), 13.7 (95\% CI 2.595.3) and $>50$, respectively) were independent predictors of ICU mortality $(\mathrm{p}<0.05)$. The risk prediction model had good calibration and discrimination, with a goodness of fit $\mathrm{p}=0.99$ and a mean area under the curve of 0.798 (95\% CI 0.724-0.872) (supplementary figure S1). A Cox model using the time to ICU mortality is reported in supplementary table S4. Lastly, the resulting logistic mixed-multivariate regression model (table 4), adjusted for centre, identified IMV (aOR 12.1 (95\% CI 3.6-60.3)), vasopressors (aOR 3.2 (95\% CI 1.1-10.1)), pneumonia (aOR 1.9 (95\% CI 0.7-5.5)) and pre-admission diagnosis of BOS stage 2, stage 3 or RAS (aOR 20.1 (95\% CI 2.4-257), 12.3 (95\% CI 2.2-95) and >50, respectively) as independent predictors for hospital mortality $(\mathrm{p}<0.05)$. Only eight $(5.2 \%)$ patients had positive DSAs before ICU readmission and this variable did not affect the models. 


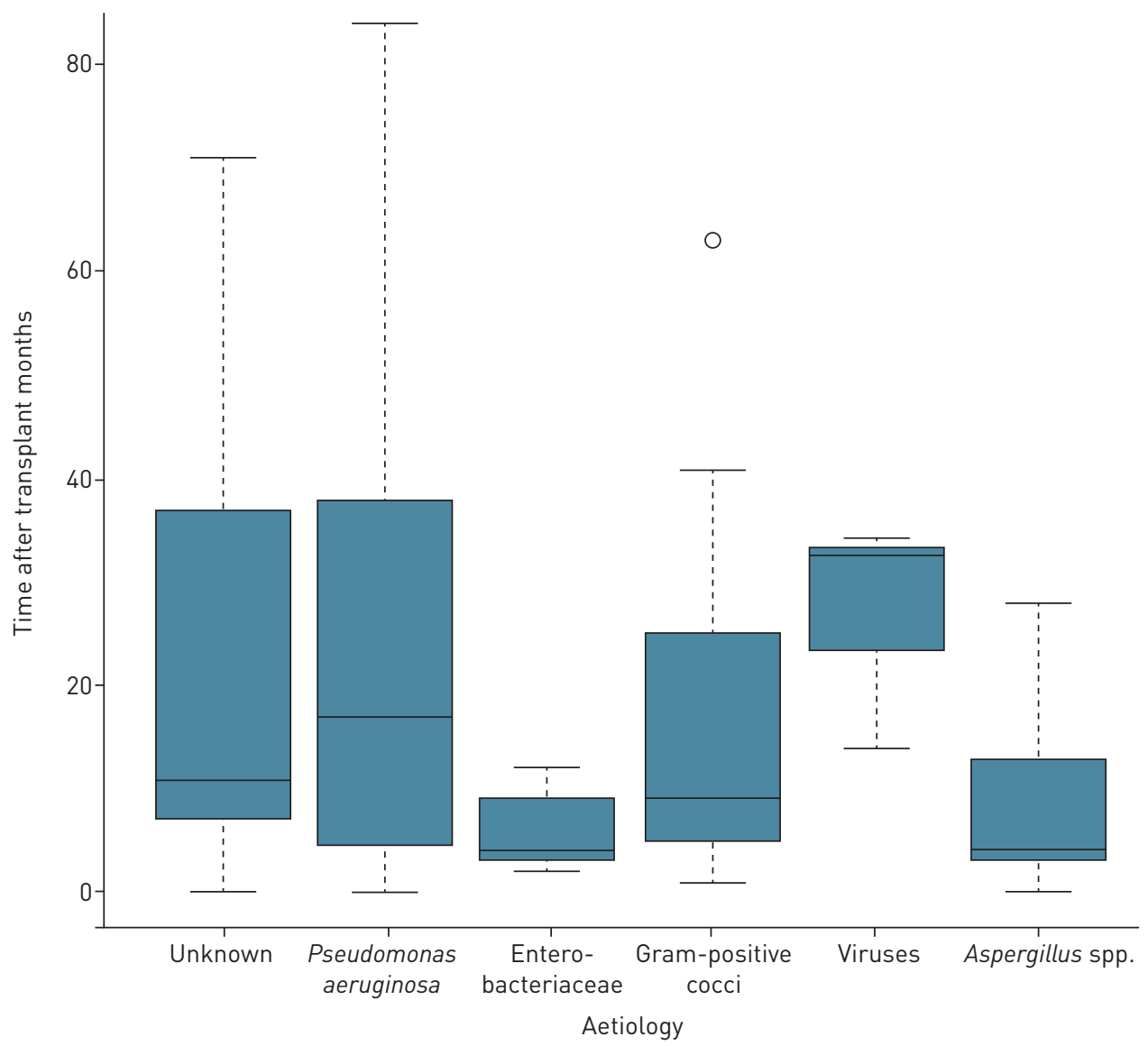

FIGURE 3 Chronology of the aetiology of the intensive care unit readmissions due to pneumonia in lung transplant recipients according to time after transplant. Data are presented as median with interquartile range (IQR) (boxes) and 1.5 IQR (whiskers); outliers are indicated.

After ICU discharge, 15 (9.8\%) patients required new ICU readmissions (twice in three subjects). These admissions occurred at a median (IQR) 46 (15-209) days after ICU discharge. Subsequent ICU readmission was associated with extremely high mortality (second ICU readmission 52.6\%; third ICU readmission 66.6\%). Causes and further details of recurrent readmissions are shown in supplementary table S5.

\section{Discussion}

We report the largest prospective, multicentre cohort study to date on lung transplant recipients in the ICU beyond the post-operative period. Importantly, bacterial pneumonia was identified as the main cause of ICU readmission (and an independent risk factor for ICU mortality), with $P$. aeruginosa being the main pathogen. Aspergillosis, P. jirovecii and respiratory virus pulmonary infections (including influenza) were unlikely causes of severe ARF. Graft rejection was relatively infrequent (only $5.2 \%$ of patients were admitted for acute allograft rejection) and presence of DSAs before ICU admission did not influence outcomes. In contrast, CLAD was identified as a significant predictor of death; survival was inversely correlated with severity of obstruction in the BOS phenotype and the RAS phenotype should be considered as an indication for palliative care.

Our data confirmed previous studies (albeit with retrospective or single-centre designs and small sample sizes) that identified ARF as the main indication for ICU readmission, followed by sepsis [12-17]. Differences between studies are detailed in supplementary table S1. However, our analysis was the first to identify an independent association of pneumonia and CLAD with mortality and the need for IMV. The intensity of allograft exposure to the external environment (and potential pathogens), organisms with high MICs, the net state of immunosuppression and ineffective clearance of secretions may explain these findings [28-30]. The fact that patients with pneumonia have poor outcomes may be because of the delay in administering adequate empirical antimicrobial therapy due to multidrug-resistant isolates [31, 32]. 


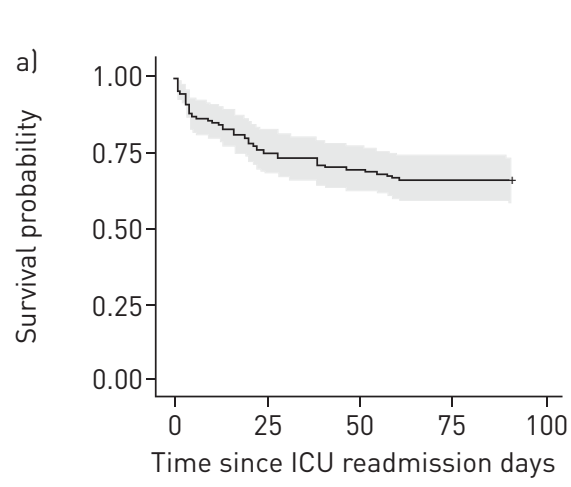

At risk $n$ :

$$
\begin{array}{lllll}
153 & 112 & 104 & 99 & 0
\end{array}
$$$$
\text { * No vasopressor - Vasopressor }
$$

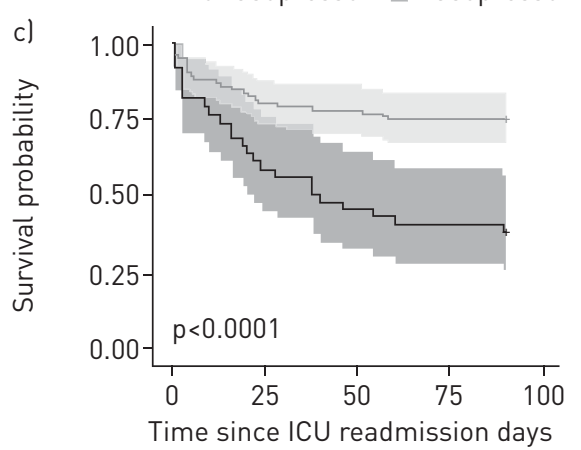

At risk $\mathrm{n}$ :

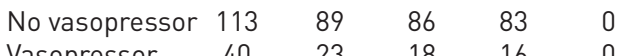

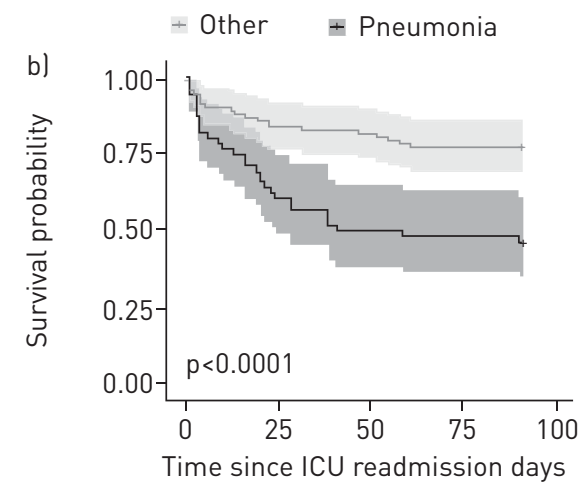

At risk $n$ :

Other

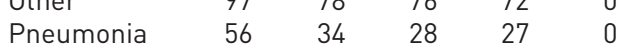

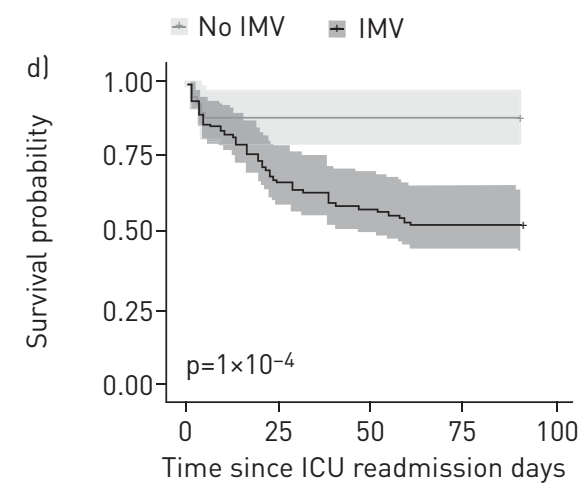

At risk $\mathrm{n}$ :

No IMV

IMV

$\begin{array}{lllll}58 & 48 & 48 & 48 & 0 \\ 40 & 64 & 56 & 51 & 0\end{array}$

FIGURE 4 Survival of lung transplant recipients after intensive care unit (ICU) readmission. IMV: invasive mechanical ventilation. a) Survival of all patients. b) Survival of patients with a diagnosis of pneumonia versus others as causes of ICU readmissions. c) Survival of patients with or without vasopressor requirement. d) Survival of patients with or without IMV requirement. Shading indicates $95 \%$ confidence intervals.

OXA-48 is a predominant mechanism of carbapenem resistance in Spain, but Klebsiella pneumoniae carbapenemase was endemic at one of the centres. Most instances of multidrug-resistant P. aeruginosa were identified in two sites (Barcelona and Santander). Over $60 \%$ of multidrug-resistant P. aeruginosa strains were highly carbapenem resistant, with $>80 \%$ only susceptible to amikacin and colistin (the only antibiotic to which all strains were susceptible). Overall, the MIC50 to meropenem was $>16 \mu \mathrm{g} \cdot \mathrm{mL}^{-1}$ and the MIC90 was $>32 \mu \mathrm{g} \cdot \mathrm{mL}^{-1}$. Improved diagnostic strategies, including new biomarkers $[33,34]$ in blood and BAL, should be implemented. Moreover, our findings emphasise the need for new antibiotics such as ceftolozane-tazobactam, due to the spread of carbapenem-resistant $P$. aeruginosa, in which early adequate treatment (mostly with colistin and aminoglycosides) does not seem to be associated with improved prognosis. Indeed, prompt pneumonia recognition and pathogen detection for targeted therapy requires the implementation of rapid diagnostic tests at the patient's bedside both to identify the microbiology and to detect resistance $[35,36]$.

The ICU mortality of $35.9 \%$ is lower than that reported by other studies (with mortality rates $>60 \%$; supplementary table S1) $[13,14]$. The differences with respect to previous results may be attributed to the heterogeneity among centres with regard to ICU readmission criteria, the small numbers of patients in previous studies, the incidence of sepsis and advances in critical care. In our cohort, CLAD showed an independent three-fold increase of risk of death. The relevance of CLAD in quality of life and as a cause of death after lung transplant has been reported in the general population [2,37], but it has not previously been identified as a prognostic factor in lung transplant recipients with ICU readmission. However, our findings highlight the importance of CLAD as a predictor of mortality in lung transplant recipients requiring 
TABLE 3 Variables associated with intensive care unit (ICU) mortality on univariate analysis

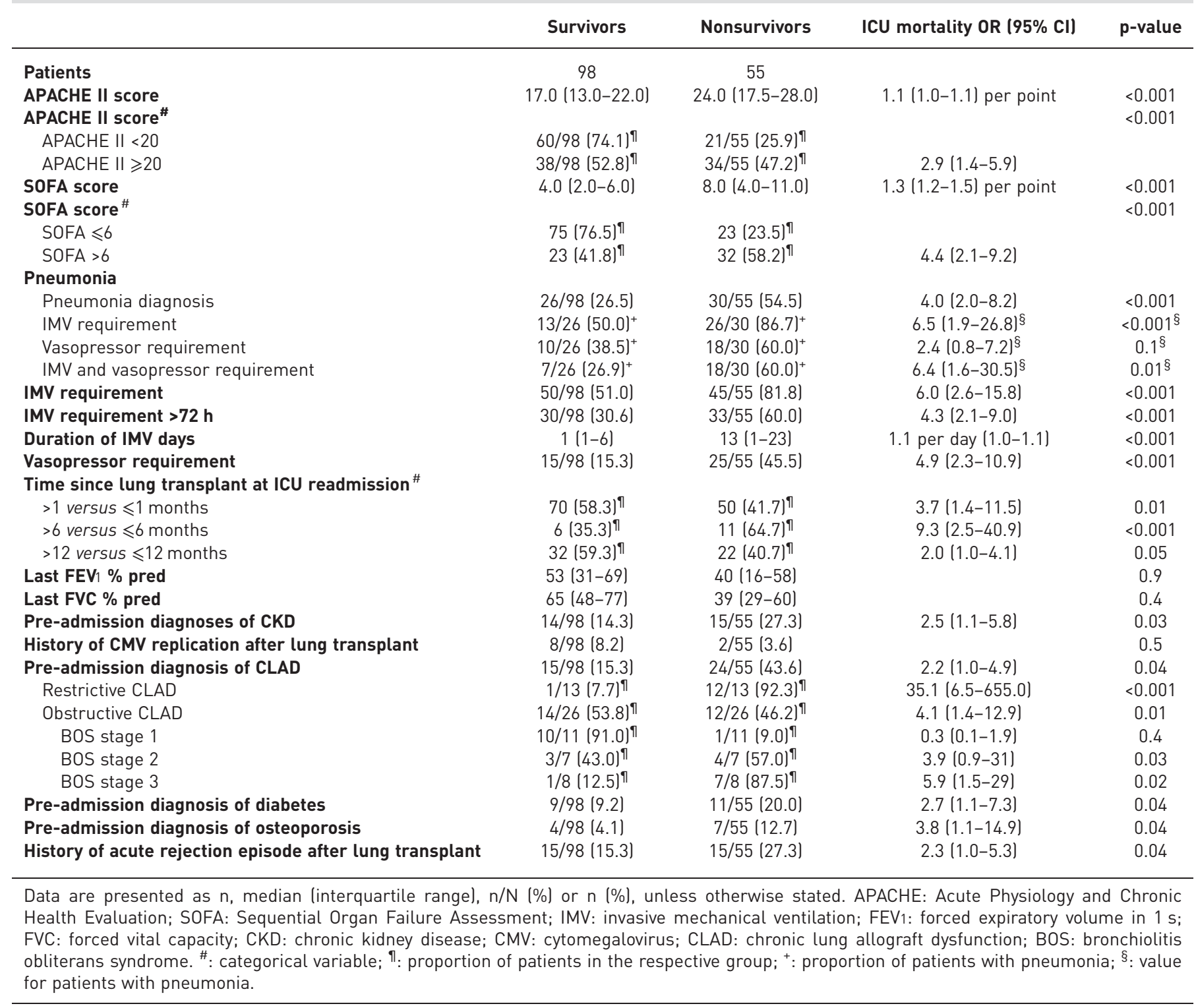

ICU readmission, taking into account CLAD phenotype and stage, with ICU survival being extremely poor for RAS $(7.7 \%)$ and BOS stage $3(12.5 \%)$ patients. Since no recipients with RAS and only one with BOS stage 3 were discharged home alive, these conditions should be considered indications for palliative care.

The variables FEV1 and FVC showed no significant differences between ICU survivors and nonsurvivors. However, in our cohort, $\sim 22 \%$ of recipients were readmitted to the ICU within the first month $(50 \%$ within 6 months) after lung transplant. This introduces a potential bias, since such a short period of time does not allow for identification of reductions in FEV1 and FVC. The current analysis has other limitations. The most important is the heterogeneity of the patients included between centres (tables 1 and 2), although the multivariate model was controlled by centre. Patients were included over a 4-year period and Sepsis 3.0 definitions cannot be applied. Data on perioperative management of these patients was not recorded, and we do not know its potential association with ICU readmission and mortality, but given the delay in readmission (median 6 months) it is unlikely to have any influence.

Our findings add information on the outcomes on lung transplant recipients and have important clinical implications. First, they emphasise the effect of CLAD, and suggest that RAS and BOS stage 3 could be considered as exclusion criteria for ICU readmission. Second, they highlight the importance of weighing the risks of high-dose corticosteroids in lung transplant recipients. As both graft failure and lung infection present a common pattern of ARF presentation but require completely opposite immunosuppressive 
TABLE 4 Variables associated with intensive care unit (ICU)/hospital mortality in multivariate regression model, clustered by investigator site

\begin{tabular}{lccc} 
Mortality predictors in multivariate analysis" & $\begin{array}{c}\text { ICU mortality } \\
\text { aOR (95\% CI) }\end{array}$ & $\begin{array}{c}\text { Hospital mortality } \\
\text { aOR (95\% CI) }\end{array}$ & p-value \\
\hline IMV requirement at ICU readmission & $8.2(2.6-34.7)$ & $12.1(3.6-60.3)$ & $<0.001$ \\
Vasopressor requirement & $3.6(1.2-11.1)$ & $3.2(1.1-10.1)$ & $<0.05$ \\
Pneumonia at ICU readmission & $2.5(1.0-7.1)$ & $1.9(0.7-5.5)$ & $<0.05$ \\
Pre-admission obstructive CLAD: BOS stage 17 & $0.5^{+}(0.1-3.5)$ & $0.4^{+}(0.1-3.0)$ & $\mathrm{NS}$ \\
Pre-admission obstructive CLAD: BOS stage 27 & $7.2(1.0-65.7)$ & $20.1(2.4-257.0)$ & $<0.01$ \\
Pre-admission obstructive CLAD: BOS stage 37 & $13.7(2.5-95.3)$ & $12.3(2.2-95.0)$ & $<0.01$
\end{tabular}

aOR: adjusted OR; IMV: invasive mechanical ventilation; CLAD: chronic lung allograft dysfunction; BOS: bronchiolitis obliterans syndrome; Ns: nonsignificant ( $p>0.05)$. \#: the noncategorical variables Acute Physiology and Chronic Health Evaluation II and Sequential Organ Failure Assessment scores were not included; ": all patients with restrictive CLAD (restrictive allograft syndrome) died, but this variable was removed from the model with the purpose of statistically evaluating the role of the different stages of BOS in the ICU/hospital mortality risk (CLAD: restrictive allograft syndrome infinite aOR with $p<0.0001$ ); ${ }^{+}$: value not statistically representative ( $p=0.5 / 0.45$ for ICU/hospital mortality, respectively).

strategies (an increase versus a decrease), a comprehensive and invasive diagnostic strategy is needed. Further studies should seek to elucidate the prognostic drivers of ARF. Finally, we stress that the causative agents of pneumonia requiring ICU readmission have changed over the past decade [38].

\section{Conclusions}

In adult lung transplant recipients requiring ICU readmission, pneumonia and CLAD (phenotype and severity of BOS) independently influenced risk of death. Other complications such as acute allograft dysfunction were uncommon.

Acknowledgements: We thank Jordi Riera (Intensive Care Dept, Vall d'Hebron University Hospital, Barcelona, Spain) for critical review, Anabel Romero (CIBERES; Barcelona, Spain) for critical review and statistical advice, and Alberto Sandiumenge (Transplant Coordination Dept, Vall d'Hebron University Hospital) for critical review. All statistical analyses were carried out by the staff of the Statistics and Bioinformatics Unit in the Vall d'Hebron Institute of Research (VHIR; Barcelona, Spain). Language assistance was provided by Fidelma Greaves (Vall d'Hebron Research Institute, Barcelona, Spain) and Michael Maudsley (Instituto de Salud Carlos III, Madrid, Spain) .

Support statement: This work was funded in part by the Instituto Salud Carlos III, Madrid and CIBERES (Fondos FEDER) (grant PI14/1296). Funding information for this article has been deposited with the Crossref Funder Registry.

Conflict of interest: None declared.

\section{References}

1 Yusen RD, Edwards LB, Dipchand AI, et al. The Registry of the International Society for Heart and Lung Transplantation: Thirty-third Adult Lung and Heart-Lung Transplant Report 2016; focus theme: primary diagnostic indications for transplant. J Heart Lung Transplant 2016; 35: 1170-1184.

2 Rello J. Lung transplant: an emerging challenge in the ICU. Med Intensiva 2012; 36: 504-505.

3 Fuehner T, Greer M, Welte T, et al. The lung transplant patient in the ICU. Curr Opin Crit Care 2012; 18: $472-478$

4 Clajus C, Blasi F, Welte T, et al. Therapeutic approach to respiratory infections in lung transplantation. Pulm Pharmacol Ther 2015; 32: 149-154.

5 Díaz-Ravetllat V, Greer M, Haverich A, et al. Significance of new lung infiltrates in outpatients after lung and heart-lung transplantation. Transpl Infect Dis 2014; 16: 359-368.

6 Yusen RD, Edwards LB, Kucheryavaya AY, et al. The Registry of the International Society for Heart and Lung Transplantation: Thirty-second Official Adult Lung and Heart-Lung Transplantation Report 2015; focus theme: early graft failure. J Heart Lung Transplant 2015; 34: 1264-1277.

7 Rello J, Bello I, de Vicente R, et al. Risk factors for mortality in 272 patients with lung transplant: a multicenter analysis of 7 intensive care units. Arch Bronconeumol 2017; 53: 421-426.

8 Verleden GM, Vos R, Vanaudenaerde B, et al. Current views on chronic rejection after lung transplantation. Transpl Int 2015; 28: 1131-1139.

9 Gauthier JM, Hachem RR, Kreisel D. Update on chronic lung allograft dysfunction. Curr Transplant Rep 2016; 3: 185-191.

10 Verleden SE, Vos R, Vanaudenaerde BM, et al. Chronic lung allograft dysfunction phenotypes and treatment. $J$ Thorac Dis 2017; 9: 2650-2659.

11 Hodge S, Holmes M, Banerjee B, et al. Posttransplant bronchiolitis obliterans syndrome is associated with bronchial epithelial to mesenchymal transition. Am J Transplant 2009; 9: 727-733. 
12 Pietrantoni C, Minai OA, Yu NC, et al. Respiratory failure and sepsis are the major causes of ICU admissions and mortality in survivors of lung transplants. Chest 2003; 123: 504-509.

13 González-Castro A, Suberviola B, Llorca J, et al. Prognosis factors in lung transplant recipients readmitted to the intensive care unit. Transplant Proc 2007; 39: 2420-2421.

14 Cohen J, Singer P, Raviv Y, et al. Outcome of lung transplant recipients requiring readmission to the intensive care unit. J Heart Lung Transplant 2011; 30: 54-58.

15 Hadjiliadis D, Steele MP, Govert JA, et al. Outcome of lung transplant patients admitted to the medical ICU. Chest 2004; 125: 1040-1045.

16 Banga A, Sahoo D, Lane CR, et al. Characteristics and outcomes of patients with lung transplantation requiring admission to the medical ICU. Chest 2014; 146: 590-599.

17 Maurer JR, Tullis E, Grossman RF, et al. Infectious complications following isolated lung transplantation. Chest 1992; 101: 1056-1059.

18 American Thoracic Society, Infectious Diseases Society of America. Guidelines for the management of adults with hospital-acquired, ventilator-associated, and healthcare-associated pneumonia. Am J Respir Crit Care Med 2005; 171: 388-416.

19 Stewart S, Fishbein MC, Snell GI, et al. Revision of the 1996 working formulation for the standardization of nomenclature in the diagnosis of lung rejection. J Heart Lung Transplant 2007; 26: 1229-1242.

20 Berry G, Burke M, Andersen C, et al. Pathology of pulmonary antibody-mediated rejection: 2012 update from the Pathology Council of the ISHLT. J Heart Lung Transplant 2013; 32: 14-21.

21 Levy MM, Fink MP, Marshall JC, et al. 2001 SCCM/ESICM/ACCP/ATS/SIS International Sepsis Definitions Conference. Crit Care Med 2003; 31: 1250-1256.

22 Christie JD, Carby M, Bag R, et al. Report of the ISHLT Working Group on Primary Lung Graft Dysfunction Part II: Definition. A consensus statement of the International Society for Heart and Lung Transplantation. J Heart Lung Transplant 2005; 24: 1454-1459.

23 Christie JD, Edwards LB, Kucheryavaya AY, et al. The Registry of the International Society for Heart and Lung Transplantation: Twenty-eighth Adult Lung and Heart-Lung Transplant Report - 2011. J Heart Lung Transplant 2011; 30: 1104-1122.

24 European Committee on Antimicrobial Susceptibility Testing. 2016. Breakpoint tables for interpretation of MICs and zone diameters, versions 1.3 and 2.0. www.eucast.org/ast_of_bacteria/previous_versions_of_documents Date last accessed: July 10, 2019.

25 Magiorakos AP, Srinivasan A, Carey RB, et al. Multidrug-resistant, extensively drug-resistant and pandrug-resistant bacteria: an international expert proposal for interim standard definitions for acquired resistance. Clin Microbiol Infect 2012; 18: 268-281.

26 Bellomo R, Ronco C, Kellum JA, et al. Acute renal failure - definition, outcome measures, animal models, fluid therapy and information technology needs: the Second International Consensus Conference of the Acute Dialysis Quality Initiative (ADQI) Group. Crit Care 2004; 8: R204-R212.

27 Levey AS, Eckardt KU, Tsukamoto $\mathrm{Y}$, et al. Definition and classification of chronic kidney disease: a position statement from Kidney Disease: Improving Global Outcomes (KDIGO). Kidney Int 2005; 67: 2089-2100.

28 Borgatta B, Gattarello S, Mazo CA, et al. The clinical significance of pneumonia in patients with respiratory specimens harbouring multidrug-resistant Pseudomonas aeruginosa: a 5-year retrospective study following 5667 patients in four general ICUs. Eur J Clin Microbiol Infect Dis 2017; 36: 2155-2163.

29 Perri A, Fumagalli R. Sepsis in transplanted patients: beyond antibiotic therapy. Transplant Proc 2008; 40: $1207-1211$

30 Awori Hayanga JW, Aboagye JK, Shigemura N, et al. Airway complications after lung transplantation: contemporary survival and outcomes. J Heart Lung Transplant 2016; 35: 1206-1211.

31 Palacio F, Reyes LF, Levine DJ, et al. Understanding the concept of health care-associated pneumonia in lung transplant recipients. Chest 2015; 148: 516-522.

32 Riera J, Caralt B, López I, et al. Ventilator-associated respiratory infection following lung transplantation. Eur Respir J 2015; 45: 726-737.

33 Riera J, Senna A, Cubero M, et al. Primary graft dysfunction and mortality following lung transplantation: role for proadrenomedullin plasma levels. Am J Transplant 2016; 16: 634-639.

34 Mazo C, Borgatta B, Pont T, et al. Procalcitonin accurately predicts lung transplant adults with low risk of pulmonary graft dysfunction and intensive care mortality. J Crit Care 2018; 44: 142-147.

35 Drick N, Seeliger B, Greer M, et al. DNA-based testing in lung transplant recipients with suspected non-viral lower respiratory tract infection: a prospective observational study. Transpl Infect Dis 2018; 20: e12811.

36 Restrepo MI, Babu BL, Reyes LF, et al. Burden and risk factors for Pseudomonas aeruginosa community-acquired pneumonia: a multinational point prevalence study of hospitalised patients. Eur Respir J 2018; 52: 1701190.

37 Künsebeck HW, Kugler C, Fischer S, et al. Quality of life and bronchiolitis obliterans syndrome in patients after lung transplantation. Prog Transplant 2007; 17: 136-141.

38 Aguilar-Guisadoa M, Givald J, Ussetti P, et al. Pneumonia after lung transplantation in the RESITRA cohort: a multicenter prospective study. Am J Transplant 2007; 7: 1989-1996. 